\title{
Ambiguity aversion and the absence of wage indexation ${ }^{\text {ts }}$
}

\author{
Sujoy Mukerji ${ }^{\mathrm{a}, \mathrm{b}, *}$, Jean-Marc Tallon ${ }^{\mathrm{c}}$ \\ ${ }^{a}$ Department of Economics, University of Oxford, Oxford, OXI 3UQ, UK \\ ${ }^{\mathrm{b}}$ University College, Oxford, OXI 4BH, UK \\ ${ }^{\mathrm{c}}$ EUREQua, CNRS, Université Paris I Panthéon-Sorbonne, 75647 Paris Cedex 13, France
}

Received 4 April 2002; received in revised form 6 January 2003; accepted 25 August 2003

\begin{abstract}
This paper analyzes optimal wage contracting assuming agents are not subjective expected utility maximizers but are, instead, ambiguity (or uncertainty) averse decision makers who maximize Choquet expected utility. We show that such agents will choose not to include any indexation coverage in their wage contracts even when inflation is uncertain, unless the perceived inflation uncertainty is high enough. Significantly, the exercise does not presume any exogenous costs (e.g., transactions costs) of including indexation links.

(C) 2004 Elsevier B.V. All rights reserved.
\end{abstract}

JEL classification: D81; J31

Keywords: Wage contracts; Nominal contracts; Indexation; Inflation; Uncertainty aversion; Knightian uncertainty; Choquet expected utility

\section{Introduction}

Wage indexation is, arguably, relatively rare except in the case of economies with persistent, highly variable inflation. For instance, in the case of US, even the proportion of workers in "major" union contracts (i.e., those covering 1000 or more

\footnotetext{
${ }^{25}$ We thank Berthold Herrendorf, Dennis Snower, Margaret Stevens, an anonymous referee and an Associate Editor for helpful suggestions and comments. Mukerji acknowledges financial support from an ESRC Research Fellowship Award. Part of this work was done while Tallon was at the Department of Applied Mathematics, Universita' Ca' Foscari di Venezia, Venezia, Italy.

*Corresponding author. Department of Economics, Oxford University, Manor Road Building, Oxford, OX1 3UQ, UK. Tel.: +44-1865-276643; fax: +44-1865-276790.

E-mail address: sujoy.mukerji@economics.ox.ac.uk (S. Mukerji).
} 
workers) with indexation clauses, while significantly correlated with the prevailing rate of inflation, has stayed below 60 per cent even during the severest episodes of inflation (Holland, 1995) and very much below that mark in less inflationary times. While formal statistics about indexation clauses in smaller union contracts or in the non-union sector are hard to find, as Card (1986) notes (footnote 1, italics added) "It is generally believed that escalation provisions are rare in the non-union sector. On this basis, perhaps no more than 10 percent of all US workers are covered by cost-ofliving provisions." That we observe so many wage contracts without any indexation coverage at all, does not accord well with the standard theory of decision making under uncertainty, assuming at least one of the parties to the employment contract, the firm or the worker, is risk averse ${ }^{1}$ and that there is at least some uncertainty about future inflation. This paper analyzes optimal wage contracting assuming agents are not subjective expected utility (SEU) maximizers but are, instead, ambiguity (or uncertainty) averse decision makers who maximize Choquet expected utility (CEU). We show that such agents will choose not to include any indexation coverage in their wage contracts unless the perceived inflation uncertainty is high enough, even in the absence of exogenous costs (e.g., transactions costs) of including indexation links.

Savage's theory (Savage, 1954) of (SEU) maximization is the received paradigm used for modeling decision-making under subjective uncertainty. A main implication of SEU is that a decision maker (DM) behaves as if her subjective assessment of likelihoods of uncertain events may be described by a precise and unique probability distribution. It is often the case, however, that a DMs knowledge about the likelihood of contingent events is consistent with more than one probability distribution. But, does how precisely she knows the relevant odds influence the choice of the typical DM? Ellsberg's classic contribution (Ellsberg, 1961) was to show that indeed it does. Ellsberg observed that imprecise information about odds affected behavior in a pervasive way: most preferred to bet on events with unambiguous rather than ambiguous odds (including, as reported by Ellsberg, Savage himself!). People adjusting their decisions depending on how well they know the relevant odds and acting with greater wariness the more vague their knowledge of the odds, is a commonly observed attitude, and has been named ambiguity (or, uncertainty) aversion. Ceteris paribus, an ambiguity averse agent will be averse to acts with payoffs that are crucially contingent on events about whose odds the agent has a relatively poor idea of, just as a risk averse agent shies away from risky acts.

Schmeidler (1989), in a pioneering contribution, developed the CEU model as one possible formalization of decision behavior that incorporates ambiguity aversion. The present paper applies this formalization. In the CEU model, as in the closely related maxmin multiple prior model presented in (Gilboa and Schmeidler, 1989), roughly put, the agent's belief is captured not by a unique probability distribution in

\footnotetext{
${ }^{1}$ Results from formal empirical research accords well with casual empiricism in showing that risk aversion is pervasive in the relevant context. Card (1986, pp. S163) reports, "the parameter estimates imply that workers are substantially more risk averse than owners, although the assumption that owners are risk neutral is rejected by a conventional test." See also, Farber (1978).
} 
the standard Bayesian fashion, but instead by a set of probabilities: the agent's belief is ambiguous in the sense that more than one probability is consistent with his knowledge. Thus not only is the outcome of an act uncertain but also the expected payoff of the action, since the payoff may be measured with respect to more than one probability. In the CEU model, it is as if an ambiguity averse DM evaluates an act by the minimum expected utility that may be (subjectively) associated with it.

Suppose that price risk comes in two types. An aggregate price risk, arising from an economy wide shock (possibly, monetary) that multiplies prices of all goods by the same factor, and specific risks, arising from demand/supply shocks to specific commodities that affects the price of a single good or a restricted class of goods. A wage contract that is set in purely nominal terms is susceptible to the aggregate price risk. On the other hand, a contract wherein wages are fully linked to an index is completely immune from aggregate price risk. Being paid in terms of an index essentially amounts to being paid units of the reference bundle of goods. However, typically, the reference bundle, such as the consumer price index (CPI), contains items that are not part of a given individual's consumption basket. Hence, an indexed wage contract unavoidably picks up the specific risks arising from fluctuations in the prices of the goods in the reference bundle that are not part of the concerned individual's consumption basket (or the firms' production set). On the other hand, a specific risk affecting only such goods would be of no concern to the agents if they were to fix their transaction purely in nominal terms. Assuming contracting parties are SEU maximizers and that at least one of them is risk averse, since both types of risks are pervasive, an optimal wage contract will typically involve partial indexation (i.e., a certain fraction of wages, strictly greater than zero, will be index linked).

With SEU maximizing agents, seemingly, the standard way to obtain an optimal wage contract that does not include any indexation coverage would be to assume some exogenous cost to including such a coverage as, for instance, was done in Gray (1978). ${ }^{2}$ However, we show in this paper that if agents are ambiguity averse with CEU preferences and if they have an ambiguous belief about specific price risks involving goods that are neither in the employee's consumption basket nor in the firm's production set, then zero indexation coverage is optimal so long as the variability of inflation is anticipated to lie within a certain bound. Significantly, the result obtains without assuming any exogenous cost of indexation (e.g., transactions or writing costs); the result also obtains whether or not the belief about the aggregate price risk is ambiguous - as we show, what is crucial is the ambiguity of belief about specific price risks.

Why might the result be of interest? There is an extensive literature, pioneered by the work of Gray (1976) and Fischer (1977), which shows that whether or not a

\footnotetext{
${ }^{2}$ Jovanovic and Ueda (1997) use a principal agent model with moral hazard and renegotiation to rationalize nominal wage contracts. In their model, the workers' effort is not observable; retail sales is the only contractible signal. They show that their model implies that indexed wage contracts are not renegotiation proof and use this finding to justify the widespread use of nominal contracts. In comparison, in our model non-indexation arises even though effort is fully observable. More significantly, unlike Jovanovic and Ueda (1997), our result relates the presence of indexation to the uncertainty about inflation.
} 
macroeconomic policy maker would wish for an extensive use of wage indexation in the economy depends on certain circumstances. The relevant sets of circumstances have largely to do with the uncertainty about different price movements. A theory which endogenously links such uncertainty to the individual's decision whether or not to use indexation, would be potentially useful in telling us whether a policy intervention (to encourage or discourage indexation) is at all necessary, and if so, what form it should take. But, whether the theory is actually useful would depend crucially on its plausibility. Even in the most developed nations, information (say, formal forecasts) about relative price movements are very hard to come by. Pick any two agents in the economy; it is inevitable that the consumer price index will include goods and services that are not part of the consumption basket of either agent. For instance, it will inevitably include housing in regions that the agents have no interest in. It is a plausible assumption that agents will have, at best, very sparse informal knowledge about possible (relative) price movements of goods and services that they never consume (or include in any production process they are involved with). Thus, if agents are typically ambiguity averse when confronted with vague information, as much experimental evidence suggests (see Camerer, 1995), then it would seem compelling (a priori) to argue that they would behave in an ambiguity averse manner when acting on beliefs about relative price movements of goods that never figure in their consumption/production plans, since these are risks about which they have the least information ${ }^{3}$. Thus our result rests on a hypothesis that is, arguably, plausible.

Finally, while there is a vast literature on ambiguity aversion (see Camerer, 1995), and indeed of the many other departures from SEU, that convincingly establishes both, the theoretical sophistication of the ideas as well as their importance in laboratory settings, this work has had little impact on the way that economics is done. In large part this is because there have been few demonstrations that economically important phenomena, that are only poorly understood on the basis of standard theory, can be understood better by using models other than the standard one (SEU). ${ }^{4}$ This paper is a contribution that hopes to go some way in bridging this gap, especially as a complement to the finding in Mukerji and Tallon (2004) which shows that ambiguity aversion (with CEU preferences) may help to explain why we see so little trade in indexed bonds. In that paper we considered a model of general market equilibrium with price taking agents, involving simultaneous clearing of financial and commodity markets and showed that a result similar to that in this paper held: if uncertainty about inflation stayed below a bound, indexed bonds would not be traded in any equilibrium, given qualifications with regard to

\footnotetext{
${ }^{3}$ It is interesting to note in this context, as reported in Shiller (1997), when asked for reasons for not opting for indexation many agents say that they are inhibited by their doubts that the government inflation numbers were valid for their individual circumstances (pp. 183, 188-190, 208). Apparently, the concern was that the official price index referred to a basket of goods that was possibly different from the individual's.

${ }^{4}$ Though, lately, the list of papers exploring the implications of the idea of ambiguity aversion in economic context, has been growing. For example, see Dow and Werlang (1992), Epstein and Wang (1994), Hansen et al., (1999), Hansen et al., (2001), Tallon (1998), Mukerji (1998), and Mukerji and Tallon (2001).
} 
ambiguous beliefs about shocks affecting relative prices. The framework in the present paper is one of bilateral contracting, not a market environment like in Mukerji and Tallon (2004). Hence, the result here does not follow from the result in the other paper. Selecting an optimal contract is, in terms of the mathematical modeling in this paper, simply a static programming problem. In the other paper the (financial) contract of interest was a general equilibrium outcome (and, as it happened, an equilibrium that is not Pareto optimal, given that the markets were incomplete). As we understand it, the same intuition explains both results, a point that is significant in so far as it shows that the intuition is robust across seemingly different environments. However, we also think, the non-market framework in this paper (which facilitates the programming solution) is useful in rendering the intuition more transparent.

The rest of the paper is organized as follows. Section 2 introduces a few necessary preliminaries about the CEU model of ambiguity aversion. Section 3 explains the model and the results. Section 4 concludes. The Appendix contains the formal proofs of the propositions.

\section{Choquet expected utility}

Let $\Omega=\left\{\omega_{i}\right\}_{i=1}^{\mathrm{N}}$ be a finite state space, and assume that the DM chooses among acts with state contingent payoffs, $f: \Omega \rightarrow \mathbb{R}$ In the CEU model (Schmeidler, 1989) an ambiguity averse DM's subjective belief is represented by a convex capacity (or a convex non-additive probability function), $v: 2^{\Omega} \rightarrow[0,1]$, such that, (i) $v(\emptyset)=0$, (ii) $v(\Omega)=1$ and, (iii) $v(X \cup Y) \geqslant v(X)+v(Y)-v(X \cap Y)$, for all $X, Y \subset \Omega$. Define the core of $v$, (notation: $\Delta(\Omega)$ is the set of all additive probability measures on $\Omega$ ):

$$
\mathscr{C}(v)=\{\psi \in \Delta(\Omega) \mid \psi(X) \geqslant v(X), \text { for all } X \subset \Omega\} .
$$

Hence, $v(X)=\min _{\psi \in \mathscr{C}(v)} \psi(X)$, may be interpreted as the lower envelope of the set $\mathscr{C}(v)$. The ambiguity of belief of an event $X$ is given by

$$
\mathscr{A}(X ; v) \equiv \max _{\psi \in \mathscr{C}(v)} \psi(X)-\min _{\psi \in \mathscr{C}(v)} \psi(X) .
$$

Like in SEU, a utility function $u: \mathbb{R}_{+} \rightarrow \mathbb{R}, u^{\prime}(\cdot) \geqslant 0$, describes DM's attitude to risk and wealth. Given a convex non-additive probability $v$, the CEU of an act is simply the minimum of all possible 'standard' expected utility values obtained by measuring the contingent utilities possible from the act with respect to each of the additive probabilities in the core of $v$

$$
\mathrm{CE}_{v} u(f)=\min _{\psi \in \mathscr{C}(v)}\left\{\sum_{\omega \in \Omega} u(f(\omega)) \psi(\omega)\right\} .
$$

The Choquet expectation of an act is just its standard expectation calculated with respect to a 'minimizing probability' corresponding to this act. Hence, in the Choquet method the DM's appraisal is not only informed by his knowledge of the 
odds but is also adjusted downwards to "correct" for the perceived imprecision of his knowledge. ${ }^{5}$ The fact that the same additive probability (in the core of relevant non-additive probability) will not in general 'minimize' the expectation for two different acts, explains why the Choquet expectations operator, unlike the standard operator, is not additive, i.e., given any acts $f$ and $g$ : $\operatorname{CE}_{v}(f)+$ $\mathrm{CE}_{v}(g) \leqslant \mathrm{CE}_{v}(f+g)$.

In our analysis, we will need to consider the independent product of capacities. The independent product of two convex capacities $v_{1}$ and $v_{2}, v_{1} \otimes v_{2}$ (suggested in Gilboa and Schmeidler, 1989), is the lower envelope of the set whose generic element is the product of two probabilities, selecting one probability from the core of one capacity and the other probability from the core of the other capacity.

\section{The optimal wage contract}

\subsection{The model}

We consider a model of a wage contract between a firm and an employee. The contract is for a transaction that would take place at a future date: the employee will deliver a unit of labor and, in turn, be paid a wage by the firm as compensation. At the point of signing the contract there is some uncertainty about what commodity prices will prevail at the future date (when the transaction takes place). Since commodity prices determine the real wage, the price uncertainty is of concern to both parties to the contract. To address the concern, the contract may set the wage wholly or partially in terms of a given commodity price index. While the particular index used is given exogenously, how much of the wage to set in nominal terms and how much to set in terms of the index is the key decision variable in the contract and the focus of our analysis.

Apart from labor, there are three goods in the model: good $x$, the only good consumed by the employee, good $y$, the output of the firm, and good $z$, which though not a part of the employee's consumption plans or the firm's production plans, does nevertheless figure in the bundle of goods whose prices are tracked by the given index. There are two sources of price uncertainty. The first source is an aggregate price shock, which multiplies all prices by the same factor. The shock consists of one of two possible realizations of this factor: high $\left(\lambda_{\mathrm{H}}\right)$ or low $\left(\lambda_{\mathrm{L}}\right)$ depending on, say, whether the money supply is high or low, respectively. The second source of price uncertainty is a (sector) specific shock, idiosyncratic to the production/consumption of $z$, which causes the price of good $z$ to be higher or lower independent of the realization of aggregate shock. The uncertain environment for the firm and the employee is summarized by four states, characterized by the realizations of the two shocks on the prices of the three goods. The four states comprising the relevant state space $\Omega=\{\omega \mid \omega=1,2,3,4\}$ and the corresponding prices are described in the

\footnotetext{
${ }^{5}$ See Mukerji (1998) (Section 1) and Mukerji (1997) for more on this intuition.
} 
following table:

$\begin{array}{ll}\text { State } & \text { Prices } \\ 1 & \left(\lambda_{\mathrm{L}} p_{x}, \lambda_{\mathrm{L}} p_{y}, \lambda_{\mathrm{L}} p_{z}^{l}\right) \\ 2 & \left(\lambda_{\mathrm{L}} p_{x}, \lambda_{\mathrm{L}} p_{y}, \lambda_{\mathrm{L}} p_{z}^{h}\right) \\ 3 & \left(\lambda_{\mathrm{H}} p_{x}, \lambda_{\mathrm{H}} p_{y}, \lambda_{\mathrm{H}} p_{z}^{l}\right) \\ 4 & \left(\lambda_{\mathrm{H}} p_{x}, \lambda_{\mathrm{H}} p_{y}, \lambda_{\mathrm{H}} p_{z}^{h}\right)\end{array}$

In the table, the price triple corresponding to each state shows, in order, the prices of $x, y$ and $z$, in that state. We use the superscripts 1 and $\mathrm{h}$, respectively, to indicate a low and a high realization of the specific shock to the price of $z$. For instance, at state 1 the aggregate shock is low, as is the specific shock (to the price of $z$ ). Consistent with our discussion, we assume $p_{z}^{\mathrm{h}} \geqslant p_{z}^{1}$ and $\lambda_{\mathrm{H}} \geqslant \lambda_{\mathrm{L}}$.

We assume that the firm produces a fixed amount of good $y$ and uses one unit of labor. Let $w_{\mathrm{N}}$ denote (part of) the wage paid by the firm in nominal units and let $w_{\mathrm{I}}$ denote the part paid in units of the indexation bundle. The indexation bundle is made up of a unit of each good. Firm's real profit (in terms of good $y$ ) in state $\omega, \pi^{\omega}$, is given by

$$
\pi^{\omega}=y-\frac{w_{\mathrm{N}}}{\lambda^{\omega} p_{y}}-\frac{\lambda^{\omega}\left(p_{x}+p_{y}+p_{z}^{\omega}\right) w_{\mathrm{I}}}{\lambda^{\omega} p_{y}}=y-\frac{w_{\mathrm{N}}}{\lambda^{\omega} p_{y}}-\frac{\left(p_{x}+p_{y}+p_{z}^{\omega}\right) w_{\mathrm{I}}}{p_{y}},
$$

where $\lambda^{1}=\lambda^{2}=\lambda_{\mathrm{L}}, \lambda^{3}=\lambda^{4}=\lambda_{\mathrm{H}}, p_{z}^{1}=p_{z}^{3}=p_{z}^{1}, p_{z}^{2}=p_{z}^{4}=p_{z}^{\mathrm{h}}$. The expression in (1) shows real profit as the residual output left after taking into account the output needed to pay the contracted wage. We will assume that the firm is risk neutral and will take that the firm's objective is to maximize its expectation (evaluated in a manner to be explained below) of this real profit. We model the objective function using real profit, rather than the nominal profit, in order that the function not be subject to money illusion. ${ }^{6}$ The firm's decision variables are $w_{\mathrm{N}}$ and $w_{\mathrm{I}}$. The firm's decision, though, does have to satisfy some additional constraints arising from considerations affecting the employee's welfare.

The employee consumes good $x$ using the wage earned. The disutility of the unit of labor to be delivered is denoted by $\bar{u}, \bar{u}>0$. The employee's utility function, a function of the consumption of $x$, is denoted $u(x)$. We assume that the employee is risk averse, hence: $u^{\prime}>0$ and $u^{\prime \prime}<0$. The employee's budget constraint in state $\omega$ is

\footnotetext{
${ }^{6}$ The "right" index to deflate the firm's profit should, arguably, comprise of prices corresponding to goods in the basket consumed by the firm's owner/employer. The results in the paper would go through if we deflated the profit with an index more "tailored" to the employer's welfare, so long as the price index used in the wage contract referred to a basket of commodities that included at least one commodity that did not directly affect the welfare of either party to the contract. In other words, the official price index, i.e., the one used in the contract, has to refer to at least one good that is neither consumed by the employee nor the employer. So, for instance, we could have deflated the firm's profit by the price of good $x$, the only good consumed by the employee, if that were the only good the employer also cared about. However, since the owner's utility does not arise directly anywhere in the model it seemed more appropriate to use the price of the only good that explicitly arises in the firm's production plans, the output, instead.
} 
given by

$$
\begin{aligned}
& \lambda^{\omega} p_{x} x^{\omega}=w_{\mathrm{N}}+\lambda^{\omega}\left(p_{x}+p_{y}+p_{z}^{\omega}\right) w_{\mathrm{I}} \\
& \Rightarrow x^{\omega}=\frac{w_{\mathrm{N}}+\lambda^{\omega}\left(p_{x}+p_{y}+p_{z}^{\omega}\right) w_{I}}{\lambda^{\omega} p_{x}} .
\end{aligned}
$$

We now turn to the specification of the firm's and the employee's beliefs.

\subsection{The case where both firm and employee are ambiguity averse}

Agents have common beliefs about the price uncertainty. To allow for the beliefs to be ambiguous, beliefs about the two types of shocks to prices are given, respectively, by two sets of (marginal) probabilities. First, consider the aggregate shock, which fixes the realization of $\lambda^{\omega}$. Let the minimum and maximum (marginal) probability on $\lambda_{\mathrm{L}}$, consistent with the agents' knowledge, be $\underline{\mu}_{\mathrm{L}}$ and $\bar{\mu}_{\mathrm{L}}$, respectively, with $\underline{\mu}_{\mathrm{L}} \leqslant \bar{\mu}_{\mathrm{L}}$. Correspondingly, the minimum and maximum (marginal) probabilities on $\lambda_{\mathrm{H}}$ are the complementary probabilities, $1-\bar{\mu}_{\mathrm{L}}$ and $1-\underline{\mu}_{\mathrm{L}}$. Next, consider the sector specific shock. Analogously, we let the minimum and maximum (marginal) probability on $p_{z}^{1}$ be $\underline{v}_{1}$ and $\bar{v}_{1}$, respectively, with $\underline{v}_{1} \leqslant \bar{v}_{1}$. The minimum and maximum probabilities define the sets of marginal probabilities. We may denote these sets of probabilities more compactly using the capacities $\mu(\cdot)$ and $v(\cdot)$, where $\mu\left(\lambda_{\mathrm{L}}\right)=\underline{\mu}_{\mathrm{L}}, \mu\left(\boldsymbol{\lambda}_{\mathrm{H}}\right)=1-\bar{\mu}_{\mathrm{L}}, v\left(p_{z}^{1}\right)=$ $\underline{v}_{1}, v\left(p_{z}^{\mathrm{h}}\right)=1-\bar{v}_{1}$. The common belief on the overall state space $\Omega$ is given by the product capacity $\mu \otimes v$. Both the firm and the employee maximize Choquet expected utility given these beliefs and the respective payoffs given the contract. Notice, the model collapses to SEU once we set $\underline{\mu}_{\mathrm{L}}=\bar{\mu}_{\mathrm{L}}$ and $\underline{v}_{1}=\bar{v}_{1}$.

The firm chooses the wage contract that maximizes the Choquet expectation of real profits $\pi^{\omega}$, given the belief $\mu \otimes v$, provided that the contracted wages are nonnegative and satisfy the employee's participation constraint. Hence, the optimal wage contract solves the program (3), below, wherein $\pi^{\omega}$ and $x^{\omega}$ are as given in (1) and (2), respectively

$$
\begin{array}{ll}
\operatorname{Max}_{w_{\mathrm{N}}, w_{\mathrm{I}}} & \mathrm{CE}_{\mu \otimes v} \pi^{\omega} \\
\text { s.t. } & \left\{\begin{array}{l}
\text { (i) } \mathrm{CE}_{\mu \otimes v} u\left(x^{\omega}\right) \geqslant \bar{u} \\
\text { (ii) } w_{\mathrm{I}} \geqslant 0 \\
\text { (iii) } w_{\mathrm{N}} \geqslant 0 .
\end{array}\right.
\end{array}
$$

Notice, the two parties "rank" every pair of states in the opposite way; if profit in state $i$ is greater than that in state $j$, consumption is greater in state $j$ as compared to state $i$. The firm is better off higher the general inflation and worse off higher the price of $z$; precisely the opposite is true for the worker. Knowing the ranking of states by each party, we may deduce which (additive) probability (from the core of the capacity describing the common belief) each party effectively "chooses" to evaluate its expectation. Let $\bar{\mu}$ represent the two point (additive) probability distribution $\left(\bar{\mu}_{\mathrm{L}}, 1-\bar{\mu}_{\mathrm{L}}\right)$ and let $\underline{\mu}$ represent the two point probability distribution $\left(\underline{\mu}_{\mathrm{L}}, 1-\underline{\mu}_{\mathrm{L}}\right)$. Similarly, let $\bar{v}$ represent the two point probability $\left(\bar{v}_{1}, 1-\bar{v}_{1}\right)$ and let $\underline{v}$ represent the two point probability $\left(\underline{v}_{1}, 1-\underline{v}_{1}\right)$. It follows, from the way the firm ranks states, that it 
is as if the firm evaluates expected profits "using" the product probability $\bar{\mu} \times \underline{v}$. To see why this is so notice that the firm, given a particular realization of the specific shock, is worse off when inflation is lower (i.e., when $\lambda^{\omega}=\lambda_{\mathrm{L}}$ rather than when $\lambda^{\omega}=\lambda_{\mathrm{H}}$ ); while, conditional on the monetary shock, the firm is worse off when price of $z$ is high $\left(\lambda^{\omega} p_{z}^{\mathrm{h}}\right)$ rather than low $\left(\lambda^{\omega} p_{z}^{1}\right)$. Next note $\bar{\mu}$, compared to $\underline{\mu}$, puts greater probability weight on $\lambda_{\mathrm{L}}$ (and less on $\lambda_{\mathrm{H}}$ ). Also, $\underline{v}$ compared to $\bar{v}$, puts greater probability weight on $p_{z}^{\mathrm{h}}$ (and less on $p_{z}^{1}$ ). Hence, the product probability in the core of the product capacity $\mu \otimes v$ which minimizes the evaluation of $\mathrm{E} \pi^{\omega}$ is $\bar{\mu} \times \underline{v}$. Analogously, the ranking of states by the employee implies that the employee evaluates expected utility "using" the probability $\underline{\mu} \times \bar{v}$, since that is the product probability in the core of the product capacity $\mu \otimes v$ which minimizes $\mathrm{E} u\left(x^{\omega}\right)$. The intuitive point is that each agent, given CEU preferences, evaluates payoffs using that probability, from the set of probabilities consistent with the common information, that makes the evaluation "robust" against the possible variation in the probabilities. Given that the agents' payoffs rank states differently, the probability distribution that meets the "robustness check" for each agent is a different one. The description of the program the contract optimizes as given in (3) may therefore be rewritten in terms of "standard" probabilities and "standard" expectation operators as follows:

$$
\begin{array}{ll}
\operatorname{Max}_{w_{\mathrm{N}}, w_{\mathrm{I}}} & \mathrm{E}_{\bar{\mu} \times \underline{\underline{\nu}} \pi^{\omega}} \\
\text { s.t. } \quad & \left\{\begin{array}{l}
\text { (i) } \mathrm{E}_{\underline{\mu} \times \bar{\nu}} u\left(x^{\omega}\right) \geqslant \bar{u} \\
\text { (ii) } w_{\mathrm{I}} \geqslant 0 \\
\text { (iii) } w_{\mathrm{N}} \geqslant 0 .
\end{array}\right.
\end{array}
$$

Being able to write the optimization program for the contract as in (4) means we may analyze the program using very standard tools, without having to refer any further to the arithmetic of Choquet integrals. An analysis of the properties of the solution to (4) yields Proposition 1. The proposition considers a situation wherein the level of inflation is uncertain (i.e., it is variable). The ambiguity of belief of the event where $p_{z}^{1}$ obtains is given by $\bar{v}_{1}-\underline{v}_{1}$, and since $v$ is a capacity on a doubleton state-space we may refer to $\bar{v}_{1}-\underline{v}_{1}$ as the ambiguity of $v, \mathscr{A}(v)$. Proposition 1 explains the relationship between the ambiguity of belief about the price of $z$ and no indexation. Part (a) shows that if ambiguity about inflation is high enough then noindexation will be optimal, so long as the variability of inflation stays below a certain bound. Part (b) of Proposition 1 shows, given that inflation is believed to be variable, ambiguity on $v$ is necessary for no-indexation to be optimal. Or, more formally

Proposition 1. Consider the optimization program posed in (4). Let $\mathscr{A}(v) \equiv \bar{v}_{1}-\underline{v}_{1}$ and let $\kappa \equiv \lambda_{\mathrm{H}} / \lambda_{\mathrm{L}}$ be such that $\kappa>1$.

(a) There exist $\overline{\mathscr{A}}$ and $\bar{\kappa}, 1>\overline{\mathscr{A}}>0$, such that if $\kappa \leqslant \bar{\kappa}$ and $\mathscr{A}(v)>\overline{\mathscr{A}}$, then $w_{\mathrm{I}}=0$ at any solution to the optimization program.

(b) Let $\left(w_{\mathrm{I}}, w_{\mathrm{N}}\right)$ be a solution to the optimization program. At such a solution $w_{\mathrm{I}}=0$ only if $\mathscr{A}(v)>0$. 
Next we discuss an intuition for the main aspects of the result. First consider a situation wherein there is some uncertainty about inflation (i.e., $\kappa>1$ ), and about the price of $z$, but there is no associated ambiguity (i.e., $\mathscr{A}(v)=\mathscr{A}(\mu)=0$ ). In other words, the common belief is described by a (standard) probability. Take a contract where the wage is set completely in nominal terms, i.e., $w_{\mathrm{I}}=0$ and consider the benefits and costs of introducing a marginal unit of indexation into this contract. Note, given the completely nominal contract, the risk averse worker is exposed to the inflation uncertainty to the fullest extent but is not exposed at all to the risk in the price of $z$. Indexation would insure the worker against the inflation risk but expose the worker to the risk involving $p_{z}$. At the margin being considered, the ratio of worker's marginal utility to the firm's marginal utility (the latter may be normalized to unity since the firm is risk neutral) is greater when $\lambda^{\omega}=\lambda_{\mathrm{H}}$ compared to when $\lambda^{\omega}=\lambda_{\mathrm{L}}$; whereas, the ratio is constant across changes in $p_{z}$, conditional on the aggregate price shock. Thus, introducing a marginal amount of indexation would enable "gains from trade" to be generated by reallocating resource from the firm to the worker when $\lambda^{\omega}=\lambda_{\mathrm{H}}$ and in the reverse direction when $\lambda^{\omega}=\lambda_{\mathrm{L}}$. Hence, introducing indexation would cause a Pareto improvement. As more indexation is introduced, the gap between ratios of marginal utilities across states differing in aggregate price shocks becomes smaller, thereby lessening the marginal gains from indexation. Simultaneously, however, the worker becomes more and more exposed to risk from $p_{z}$. Resources are reallocated towards the worker when $p_{z}$ is high and away from the worker when $p_{z}$ is low. Since resources are allocated towards worker when the ratio of the worker's to the firm's marginal utility is lower, there is a consequent "loss from trade". This is the marginal cost of indexation, which rises as more indexation is introduced. As usual, optimal indexation occurs at the point the marginal costs equal the marginal benefits. However, at the initial margin, where $w_{\mathrm{I}}=0$, the marginal benefit is positive while the marginal cost is zero. Hence, the optimal amount of indexation must be strictly positive.

Next, suppose that $\mathscr{A}(v)>0$ but $\mathscr{A}(\mu)=0$. There is no ambiguity of belief about the inflation uncertainty but there is ambiguity about the uncertainty concerning the price of $z$. Consider the same thought exercise as in the preceding paragraph, that is the benefits and costs of introducing a marginal amount of indexation at the margin where $w_{\mathrm{I}}=0$, all wage is nominal and $\kappa>1$. Exactly as before, introducing a marginal amount of indexation would enable gains from trade to be generated by reallocating resource from the firm to the worker when $\lambda^{\omega}=\lambda_{\mathrm{H}}$ and in the reverse direction when $\lambda^{\omega}=\lambda_{\mathrm{L}}$. But now, unlike in the previous case, the marginal cost is positive even at this initial margin. To understand this, first note that even though ratios of marginal utilities do not differ across $p_{z}$ shocks, the firm evaluates the expected value of the transfer by attaching a higher probability weight to the event where $p_{z}$ is higher. But the worker evaluates expectation by attaching a higher weight where $p_{z}$ is lower. Effectively, then, because of the use of different probability weights, the ratios of marginal valuations differ across the different $p_{z}$ shocks. Also, the transfer is in the "perverse" direction, i.e., from the firm when its marginal valuation is relatively higher. Thus unless the marginal gains are high enough, i.e., unless the risk due to aggregate price shock is big enough, the marginal costs swamp 
marginal benefits even at the initial margin and it is optimal to not introduce any indexation at all.

Finally, to see that it is ambiguity about the price of $z$ which is crucial and that ambiguity about the aggregate price shock does not matter for the no indexation result, reconsider the thought exercise with $\mathscr{A}(v)=0$ but $\mathscr{A}(\mu)>0$. In this case, the marginal costs work out exactly as in the case with $\mathscr{A}(v)=\mathscr{A}(\mu)=0$. But marginal benefits (of indexation) are actually greater! Now, the firm evaluates expected transfers by putting a relatively low probability weight when $\lambda^{\omega}=\lambda_{\mathrm{H}}$ and the worker evaluates expected utility (or consumption) by putting a relatively low probability weight when $\lambda^{\omega}=\lambda_{\mathrm{L}}$. Exactly as before, introducing a marginal amount of indexation would enable gains from trade to be generated by reallocating resource from the firm to the worker when $\lambda^{\omega}=\lambda_{\mathrm{H}}$ and in the reverse direction when $\lambda^{\omega}=\lambda_{\mathrm{L}}$, but now the gains are greater because not only are the ratios of marginal utilities aligned the "right" way but the disparity in probability weights is also such that the "giver" values the resource less than the "receiver".?

A final point about the logic of the result that is worth clarifying is that it obtains whether or not the principal, i.e., the employer is ambiguity averse. To this end we consider, in the following subsection the case where the firm has probabilistic belief (i.e., is ambiguity neutral) and show that the result holds in this case too.

\subsection{The case of an ambiguity neutral firm}

It might be argued that firms are better informed than workers and may have more objective information, or at the least, consider and process information more objectively. Hence, it is worth considering how the model works out if we were to assume that only the worker is ambiguity averse while the firm is an expected value maximizer with a probabilistic belief. (We assume, as before, that the firm is risk neutral.) In what follows we reconsider the model presented in the last section by amending beliefs accordingly. More specifically, we assume that the firm "knows" the "true" probability governing the states, while the worker contemplates a set of probabilities centered around this true probability. Thus it is as if the firm and the worker's beliefs are informed of the same empirical regularity, but while the firm's information about the regularity is relatively precise the worker's information is more ambiguous.

Accordingly, we assume the firm has probabilistic beliefs $(m, 1-m)$ on $\left(\lambda_{\mathrm{L}}, \lambda_{\mathrm{H}}\right)$ and $(n, 1-n)$ on $\left(p_{z}^{1}, p_{z}^{\mathrm{h}}\right)$ with $1>m>0$ and $1>n>0$. (With slight abuse of notation, $m$ and $n$ will also be used to denote these (two point) probability distributions.) The worker is not sure that the true probabilities are $m$ and $n$. He entertains as possible two sets of probabilities, each symmetrically "centered" around one of these two reference distributions. More formally, the employee's belief on the states $\lambda_{\mathrm{L}}, \lambda_{\mathrm{H}}$, is

\footnotetext{
${ }^{7}$ In this special case, where $\mathscr{A}(v)=0$ but $\mathscr{A}(\mu)>0$, if $\mathscr{A}(\mu)$ is large enough, it is possible that $w_{\mathrm{N}}=0$ in the optimal solution. However, this is an artefact of a simplifying assumption that we made, that the worker has no obligation (like house rent) that is contracted (at least partly) in nominal terms, or any other source of income (returns from savings) that is (at least partly) set in nominal terms.
} 
given by the set $\mathscr{C}\left(\lambda_{\mathrm{L}}, \lambda_{\mathrm{H}}\right)$ specified as follows, where $\Delta$ denotes the two-dimensional unit simplex and the parameter $\mathscr{A} \in(0,1)$

$$
\begin{aligned}
\mathscr{C}\left(\boldsymbol{\lambda}_{\mathrm{L}}, \lambda_{\mathrm{H}}\right) \equiv & \left\{\left(m^{\prime}\left(\boldsymbol{\lambda}_{\mathrm{L}}\right), m^{\prime}\left(\boldsymbol{\lambda}_{\mathrm{H}}\right)\right) \in \triangle \mid m^{\prime}\left(\boldsymbol{\lambda}_{\mathrm{L}}\right) \geqslant(1-\mathscr{A}) m\left(\lambda_{\mathrm{L}}\right) ;\right. \\
& \left.m^{\prime}\left(\boldsymbol{\lambda}_{\mathrm{H}}\right) \geqslant(1-\mathscr{A}) m\left(\lambda_{\mathrm{H}}\right)\right\} .
\end{aligned}
$$

Similarly, the employee's beliefs on the states $p_{z}^{1}, p_{z}^{\mathrm{h}}$ is given by the set $\mathscr{C}\left(p_{z}^{1}, p_{z}^{\mathrm{h}}\right)$ specified as follows:

$$
\begin{aligned}
\mathscr{C}\left(p_{z}^{1}, p_{z}^{\mathrm{h}}\right) \equiv & \left\{\left(n^{\prime}\left(p_{z}^{1}\right), n^{\prime}\left(p_{z}^{\mathrm{h}}\right)\right) \in \triangle \mid n^{\prime}\left(p_{z}^{1}\right) \geqslant(1-\mathscr{A}) n\left(p_{z}^{1}\right) ;\right. \\
& \left.n^{\prime}\left(p_{z}^{\mathrm{h}}\right) \geqslant(1-\mathscr{A}) n\left(p_{z}^{\mathrm{h}}\right)\right\} .
\end{aligned}
$$

Let us now represent the beliefs of the ambiguity averse worker given in (5) and (6) in terms of the capacities $\mu$ and $v$ where $\mu\left(\lambda_{\mathrm{L}}\right)=\underline{\mu}_{\mathrm{L}}, \mu\left(\lambda_{\mathrm{H}}\right)=1-\bar{\mu}_{\mathrm{L}}, v\left(p_{z}^{1}\right)=\underline{v}_{1}$, $v\left(p_{z}^{\mathrm{h}}\right)=1-\bar{v}_{1}$. The capacities take the following simple form:

$$
\begin{aligned}
& \mu\left(\lambda_{\mathrm{L}}\right)=(1-\mathscr{A}) m\left(\lambda_{\mathrm{L}}\right), \mu\left(\lambda_{\mathrm{H}}\right)=(1-\mathscr{A}) m\left(\lambda_{\mathrm{H}}\right), \\
& v\left(p_{z}^{1}\right)=(1-\mathscr{A}) n\left(p_{z}^{1}\right), v\left(p_{z}^{\mathrm{h}}\right)=(1-\mathscr{A}) n\left(p_{z}^{\mathrm{h}}\right) .
\end{aligned}
$$

Thus, the worker allows for (symmetric) deviation from the reference probability by a factor $1-\mathscr{A}$. Note that $\mathscr{A}$ is the ambiguity parameter in this case since

$$
\mathscr{A}(v) \equiv \bar{v}_{1}-\underline{v}_{1}=\mathscr{A}=\mathscr{A}(\mu) \equiv \bar{\mu}_{\mathrm{L}}-\underline{\mu}_{\mathrm{L}} .
$$

The value $\mathscr{A}=1$ corresponds to extreme pessimism while $\mathscr{A}=0$ corresponds to probabilistic beliefs, i.e., ambiguity neutrality. As before, the employee's belief on $\Omega$ is given by $\mu \otimes v .^{8}$

As in the previous section, the firm chooses the wage contract that maximizes the Choquet expectation of real profits $\pi^{\omega}$, given the belief $m \times n$, provided that the contracted wages are non-negative and satisfy the employee's participation constraint. Hence, the optimal wage contract solves program (8), below, wherein $\pi^{\omega}$ and $x^{\omega}$ are as given in (1) and (2), respectively

$$
\begin{array}{ll}
\operatorname{Max}_{w_{\mathrm{N}}, w_{\mathrm{I}}} & \mathrm{CE}_{m \times n} \pi^{\omega} \\
\text { s.t. } & \left\{\begin{array}{l}
\text { (i) } \mathrm{CE}_{\mu \otimes v} u\left(x^{\omega}\right) \geqslant \bar{u} \\
\text { (ii) } w_{\mathrm{I}} \geqslant 0 \\
\text { (iii) } w_{\mathrm{N}} \geqslant 0 .
\end{array}\right.
\end{array}
$$

Given the worker's ranking of the states, the description of the program the contract optimizes as given in (8) may therefore be rewritten in terms of "standard" probabilities and "standard" expectation operators as follows, where $\underline{\mu}$ denotes the probability vector $\left(\underline{\mu}_{\mathrm{L}}, 1-\underline{\mu}_{\mathrm{L}}\right)$ on $\left(\lambda_{\mathrm{L}}, \lambda_{\mathrm{H}}\right)$ and $\bar{v}$ denotes the probability

\footnotetext{
${ }^{8}$ A more general way of specifying the employee's beliefs would have been to assume that they are given by any convex capacity whose core contains the probabilistic beliefs of the firm. This much commonality of beliefs between the firm and the employee is all that is needed to obtain the formal proposition. The assumption that the sets of belief are symmetric/"centered", as in (5) and (6), enables us to avoid the notational clutter the more general form will necessarily entail.
} 
vector $\left(\bar{v}_{1}, 1-\bar{v}_{1}\right)$ on $\left(p_{z}^{1}, p_{z}^{\mathrm{h}}\right)$

$\operatorname{Max}_{w_{\mathrm{N}}, w_{\mathrm{I}}} \mathrm{E}_{m \times n} \pi^{\omega}$

s.t. $\quad\left\{\begin{array}{l}\text { (i) } \mathrm{E}_{\underline{\mu} \times \bar{v}} u\left(x^{\omega}\right) \geqslant \bar{u} \\ \text { (ii) } w_{\mathrm{I}} \geqslant 0 \\ \text { (iii) } w_{\mathrm{N}} \geqslant 0 .\end{array}\right.$

Proposition 2. Consider the optimization program posed in (9). Let $\kappa \equiv \lambda_{\mathrm{H}} / \lambda_{\mathrm{L}}$ be such that $\kappa>1$ and let $\mathscr{A}$ be the ambiguity parameter as described in (7).

(a) There exist $\overline{\mathscr{A}}$ and $\bar{\kappa}, 1>\overline{\mathscr{A}}>0$, such that if $\kappa \leqslant \bar{\kappa}$ and $\mathscr{A}>\overline{\mathscr{A}}$, then $w_{\mathrm{I}}=0$ at any solution to the optimization program.

(b) Let $\left(w_{\mathrm{I}}, w_{\mathrm{N}}\right)$ be a solution to the optimization program. At such a solution, $w_{\mathrm{I}}=0$ only if $\mathscr{A}>0$.

Hence, Proposition 2 establishes that the results proved in the previous subsection are robust to modification of the firm's attitude towards ambiguity. The intuition for this case is almost identical to the case where both the firm and employee had a common ambiguous belief. To see this, think again of the intuition for the case where $\mathscr{A}(v)>0$ but $\mathscr{A}(\mu)=0$. The crucial point there was that the marginal cost of introducing indexation was positive even at the initial margin, where $w_{\mathrm{I}}=0$. This was so because the firm evaluates expected value by attaching a higher probability weight to the event where $p_{z}$ is higher while the employee attaches a higher weight where $p_{z}$ is lower. However, this difference in the way expectations are evaluated by the firm and the employee is true even with our new specification of beliefs. Hence, the old intuition still applies. Finally, it is worth reiterating the point mentioned in footnote 8: the same result holds whenever the employee's beliefs are ambiguous and encompass the probabilistic beliefs of the firm.

\section{Conclusion}

Thus, we see that ambiguity averse agents with CEU preferences will write completely unindexed wage contracts even when inflation is uncertain, as long as the uncertainty about inflation is not too high. The crucial link in the syllogism is the ambiguous uncertainty about relative price shocks. More precisely, it is the ambiguity about price shocks specific to those goods which, while included in the indexation bundle, do not otherwise concern the parties to the contract. ${ }^{9}$ To the

\footnotetext{
${ }^{9}$ In the analysis the indexation bundle was exogenously given. This can be relaxed considerably without affecting the result. The restriction that is necessary is that none of the available indexation bundles are completely relation specific. In other words, if in any index that contracting parties may choose to refer to is such that at least one good included in the index is not of interest to either party, then the no-indexation result goes through.
} 
macroeconomic policy maker, economy wide indexation is a " bad thing" when relative price shocks (or, real shocks, as opposed to monetary shocks) are significant. The result in this paper suggests that if the variability of relative prices is high, decision making at the "microlevel" will result in less indexation. Thus there is a congruence between micromotives and macropolicy imperatives. Of course, if one would want to promote indexation for other reasons, one way to do that, if one believed in the theory presented here, would be to create price indices that are more local and specialized. The result in the paper also seems to imply that indexing provisions are likely to appear in longer term contracts, since inflation is likely to be more variable over the longer term. ${ }^{10}$ Casual empiricism suggests, large union contracts typically involve longer terms than small union/non-union contracts. Perhaps, this explains why it is the larger union contracts which have indexation clauses.

\section{Appendix A}

Proof of Proposition 1. Consider the first order condition (FOC) for the maximization program in (4). Let $\gamma_{u}, \gamma_{\mathrm{I}}, \gamma_{\mathrm{N}}$ be the Lagrange multipliers associated with the constraints (i), (ii), (iii).

(a) We look at the FOC at $w_{\mathrm{I}}=0$. At this point, we know that $w_{\mathrm{N}}$ has to be strictly positive to satisfy the participation constraint (i), and hence the associated multiplier, $\gamma_{\mathrm{N}}$, is zero. Thus, the FOC can be written as follows:

$$
\left\{\begin{array}{l}
\mathrm{E}_{\bar{\mu} \times \underline{v}}\left[\frac{1}{\lambda^{\omega} p_{y}}\right]=\gamma_{u} \mathrm{E}_{\underline{\mu} \times \bar{v}}\left[\frac{1}{\lambda^{\omega} p_{x}} u^{\prime}\left(x^{\omega}\right)\right] \\
\mathrm{E}_{\bar{\mu} \times \underline{v}}\left[\frac{\left(p_{x}+p_{y}+p_{z}^{\omega}\right)}{p_{y}}\right]=\gamma_{u} \mathrm{E}_{\underline{\mu} \times \bar{v}}\left[\frac{p_{x}+p_{y}+p_{z}^{\omega}}{p_{x}} u^{\prime}\left(x^{\omega}\right)\right]+\gamma_{\mathrm{I}}
\end{array}\right.
$$

We may rearrange terms in (10), to get (11), below

$$
\left\{\begin{array}{l}
\gamma_{u}=\frac{\mathrm{E}_{\bar{\mu} \times \underline{\underline{v}}}\left[1 / \lambda^{\omega} p_{y}\right]}{\mathrm{E}_{\underline{\mu} \times \bar{v}}\left[1 / \lambda^{\omega} p_{x} u^{\prime}\left(x^{\omega}\right)\right]} \\
\gamma_{\mathrm{I}}=-\gamma_{u} \mathrm{E}_{\underline{\mu} \times \bar{v}}\left[\frac{p_{x}+p_{y}+p_{z}^{\omega}}{p_{x}} u^{\prime}\left(x^{\omega}\right)\right]+\mathrm{E}_{\bar{\mu} \times \underline{\underline{v}}}\left[\frac{\left(p_{x}+p_{y}+p_{z}^{\omega}\right)}{p_{y}}\right] .
\end{array}\right.
$$

We now study the sign of $\gamma_{\mathrm{I}}$. Substituting the expression for $\gamma_{u}$ obtained in the first equality in (11) into the second equality in (11) we obtain the following expression for $\gamma_{I}$.

\footnotetext{
${ }^{10}$ This hypothesis was also analyzed in Gray (1978). That analysis showed that if there was a exogenous, fixed per period cost of including indexation clauses, there will exist a "cut-off" contract length above which index clauses will be incorporated and below which they will not. The present paper shows how such a cost may arise endogenously, due to ambiguity aversion.
} 


$$
\begin{aligned}
\gamma_{\mathrm{I}}= & -\frac{\mathrm{E}_{\bar{\mu} \times \underline{\underline{v}}}\left[1 / \lambda^{\omega} p_{y}\right]}{\mathrm{E}_{\underline{\mu} \times \bar{v}}\left[1 /\left(\lambda^{\omega} p_{x}\right) u^{\prime}\left(x^{\omega}\right)\right]} \mathrm{E}_{\underline{\mu} \times \bar{v}}\left[\frac{p_{x}+p_{y}+p_{z}^{\omega}}{p_{x}} u^{\prime}\left(x^{\omega}\right)\right] \\
& +\mathrm{E}_{\bar{\mu} \times \underline{v}}\left[\frac{\left(p_{x}+p_{y}+p_{z}^{\omega}\right)}{p_{y}}\right] .
\end{aligned}
$$

Since $w_{\mathrm{I}}=0$ implies that $p_{z}^{\omega}$ and $x^{\omega}$ are independent, the expression on the r.h.s. of (12) may be simplified to yield

$$
\begin{aligned}
\gamma_{\mathrm{I}}= & -\frac{\left(1 / p_{y}\right) \mathrm{E}_{\bar{\mu} \times \underline{\underline{v}}}\left[1 / \lambda^{\omega}\right]}{\mathrm{E}_{\underline{\mu} \times \bar{v}}\left[1 /\left(\lambda^{\omega}\right) u^{\prime}\left(x^{\omega}\right)\right]} \mathrm{E}_{\underline{\mu} \times \bar{v}}\left[p_{x}+p_{y}+p_{z}^{\omega}\right] \mathrm{E}_{\underline{\mu} \times \bar{v}}\left[u^{\prime}\left(x^{\omega}\right)\right] \\
& +\left(\frac{1}{p_{y}}\right) \mathrm{E}_{\bar{\mu} \times \underline{v}}\left[\left(p_{x}+p_{y}+p_{z}^{\omega}\right)\right] .
\end{aligned}
$$

Rearranging terms on r.h.s. of the equality displayed above, it can be seen that the sign of $\gamma_{\mathrm{I}}$ is the same as that of (14), below

$$
-\frac{\mathrm{E}_{\bar{\mu} \times \underline{\underline{v}}}\left[1 / \lambda^{\omega}\right] \mathrm{E}_{\underline{\mu} \times \bar{v}}\left[u^{\prime}\left(x^{\omega}\right)\right]}{\mathrm{E}_{\underline{\mu} \times \bar{v}}\left[\left(1 / \lambda^{\omega}\right) u^{\prime}\left(x^{\omega}\right)\right]}+\frac{\mathrm{E}_{\bar{\mu} \times \underline{\underline{v}}}\left[\left(p_{x}+p_{y}+p_{z}^{\omega}\right)\right]}{\mathrm{E}_{\underline{\mu} \times \bar{v}}\left[p_{x}+p_{y}+p_{z}^{\omega}\right]} .
$$

Now, observe that if $\lambda_{\mathrm{L}}=\lambda_{\mathrm{H}}$,

$$
\frac{\mathrm{E}_{\bar{\mu} \times \underline{\underline{v}}}\left[1 / \lambda^{\omega}\right] \mathrm{E}_{\underline{\mu} \times \bar{v}}\left[u^{\prime}\left(x^{\omega}\right)\right]}{\mathrm{E}_{\underline{\mu} \times \bar{v}}\left[\left(1 / \lambda^{\omega}\right) u^{\prime}\left(x^{\omega}\right)\right]}=1 .
$$

Furthermore, $\bar{v}_{1}-\underline{v}_{1}$

$$
\frac{\mathrm{E}_{\bar{\mu} \times \underline{v}}\left[\left(p_{x}+p_{y}+p_{z}^{\omega}\right)\right]}{\mathrm{E}_{\underline{\mu} \times \bar{v}}\left[p_{x}+p_{y}+p_{z}^{\omega}\right]}=\frac{p_{x}+p_{y}+\underline{v}_{1} p_{z}^{1}+\left(1-\underline{v}_{1}\right) p_{z}^{\mathrm{h}}}{p_{x}+p_{y}+\left(1-\bar{v}_{1}\right) p_{z}^{1}+\bar{v}_{1} p_{z}^{\mathrm{h}}} .
$$

Hence, if $\underline{v}_{1}=0$ and $\bar{v}_{1}=1$, then

$$
\frac{\mathrm{E}_{\bar{\mu} \times \underline{v}}\left[\left(p_{x}+p_{y}+p_{z}^{\omega}\right)\right]}{\mathrm{E}_{\underline{\mu} \times \bar{v}}\left[p_{x}+p_{y}+p_{z}^{\omega}\right]}=\frac{p_{x}+p_{y}+p_{z}^{\mathrm{h}}}{p_{x}+p_{y}+p_{z}^{1}}>1 .
$$

So, in the extreme case in which $\lambda_{\mathrm{L}}=\lambda_{\mathrm{H}}$ and $\underline{v}_{1}=0$ and $\bar{v}_{1}=1$,

$$
\begin{gathered}
-\frac{\mathrm{E}_{\bar{\mu} \times \underline{\underline{v}}}\left[1 / \lambda^{\omega}\right] \mathrm{E}_{\underline{\mu} \times \bar{v}}\left[u^{\prime}\left(x^{\omega}\right)\right]}{\mathrm{E}_{\underline{\mu} \times \bar{v}}\left[\left(1 / \lambda^{\omega}\right) u^{\prime}\left(x^{\omega}\right)\right]}+\frac{\mathrm{E}_{\bar{\mu} \times \underline{\underline{v}}}\left[\left(p_{x}+p_{y}+p_{z}^{\omega}\right)\right]}{\mathrm{E}_{\underline{\mu} \times \bar{v}}\left[p_{x}+p_{y}+p_{z}^{\omega}\right]} \\
=-1+\frac{\mathrm{E}_{\bar{\mu} \times \underline{v}}\left[\left(p_{x}+p_{y}+p_{z}^{\omega}\right)\right]}{\mathrm{E}_{\underline{\mu} \times \bar{v}}\left[p_{x}+p_{y}+p_{z}^{\omega}\right]}>0 .
\end{gathered}
$$

As a result, in this extreme case, the FOC are satisfied at $w_{\mathrm{I}}=0$ and $w_{\mathrm{N}}$ such that the participation constraint of the household is just met, i.e., for these values of wages, we can find non-negative Lagrange multipliers such that the FOC of the maximization problem are satisfied. Now, by continuity, the expression on the 1.h.s. of (15) remains positive whenever $\lambda_{\mathrm{L}}$ is close to $\lambda_{\mathrm{H}}$ and $\varepsilon>\underline{v}_{l}>0$ and $1-\varepsilon^{\prime}<\bar{v}_{1}<1$, for $\varepsilon, \varepsilon^{\prime}$ small enough.

Given that $u(\cdot)$ is strictly concave, the programming problem (4) admits a unique solution. Hence, since we exhibited a solution to (4) at which $w_{\mathrm{I}}=0$, the assertion in part (a) of the proposition follows. 
(b) Let us suppose that $w_{\mathrm{I}}=0$ and $\mathscr{A}(v)=0$, i.e., $\bar{v}_{1}=\underline{v}_{1} \equiv \tilde{v}$. Now, following on from (14), substituting in $\tilde{v}$ in place of $\bar{v}_{1}$ and $\underline{v}_{1}$, we see that the sign of $\gamma_{\mathrm{I}}$ is the same as that of the expression below

$$
\begin{aligned}
&- \frac{\mathrm{E}_{\bar{\mu} \times \tilde{v}}\left[1 / \lambda^{\omega}\right] \mathrm{E}_{\underline{\mu} \times \tilde{v}}\left[u^{\prime}\left(x^{\omega}\right)\right]}{\mathrm{E}_{\underline{\mu} \times \tilde{v}}\left[\left(1 / \lambda^{\omega}\right) u^{\prime}\left(x^{\omega}\right)\right]}+\frac{\mathrm{E}_{\bar{\mu} \times \tilde{v}}\left[\left(p_{x}+p_{y}+p_{z}^{\omega}\right)\right]}{\mathrm{E}_{\underline{\mu} \times \tilde{v}}\left[p_{x}+p_{y}+p_{z}^{\omega}\right]} \\
&=-\frac{\mathrm{E}_{\bar{\mu} \times \tilde{v}}\left[1 / \lambda^{\omega}\right] \mathrm{E}_{\underline{\mu} \times \tilde{v}}\left[u^{\prime}\left(x^{\omega}\right)\right]}{\mathrm{E}_{\underline{\mu} \times \tilde{v}}\left[\left(1 / \lambda^{\omega}\right) u^{\prime}\left(x^{\omega}\right)\right]}+1,
\end{aligned}
$$

since,

$$
\mathrm{E}_{\bar{\mu} \times \tilde{v}}\left[\left(p_{x}+p_{y}+p_{z}^{\omega}\right)\right]=\mathrm{E}_{\tilde{v}}\left[\left(p_{x}+p_{y}+p_{z}^{\omega}\right)\right]=\mathrm{E}_{\underline{\mu} \times \tilde{v}}\left[p_{x}+p_{y}+p_{z}^{\omega}\right] .
$$

Now, we want to show that $\mathrm{E}_{\bar{\mu} \times \tilde{v}}\left[1 / \lambda^{\omega}\right] \mathrm{E}_{\mu \times \tilde{v}}\left[u^{\prime}\left(x^{\omega}\right)\right] / \mathrm{E}_{\underline{\mu} \times \tilde{v}}\left[\left(1 / \lambda^{\omega}\right) u^{\prime}\left(x^{\omega}\right)\right]>1$. Since, by hypothesis $w_{\mathrm{I}}=0$, we get that $x^{\omega}=w_{\mathrm{N}} / \bar{\lambda}^{\omega} p_{x}$. Hence, $1 / \lambda^{\omega}$ and $u^{\prime}\left(x^{\omega}\right)$ are negatively correlated, which implies,

$$
\mathrm{E}_{\underline{\mu} \times \tilde{v}}\left[\frac{1}{\lambda^{\omega}}\right] \mathrm{E}_{\underline{\mu} \times \tilde{v}}\left[u^{\prime}\left(x^{\omega}\right)\right]>\mathrm{E}_{\underline{\mu} \times \tilde{v}}\left[\frac{1}{\lambda^{\omega}} u^{\prime}\left(x^{\omega}\right)\right] .
$$

But notice,

$$
\mathrm{E}_{\bar{\mu} \times \tilde{v}}\left[\frac{1}{\lambda^{\omega}}\right]=\mathrm{E}_{\bar{\mu}}\left[\frac{1}{\lambda^{\omega}}\right] \geqslant \mathrm{E}_{\underline{\mu}}\left[\frac{1}{\lambda^{\omega}}\right]=\mathrm{E}_{\underline{\mu} \times \tilde{v}}\left[\frac{1}{\lambda^{\omega}}\right] .
$$

Hence $\mathrm{E}_{\bar{\mu} \times \tilde{v}}\left[1 / \lambda^{\omega}\right] \mathrm{E}_{\underline{\mu} \times \tilde{v}}\left[u^{\prime}\left(x^{\omega}\right)\right] / \mathrm{E}_{\underline{\mu} \times \tilde{v}}\left[\left(1 / \lambda^{\omega}\right) u^{\prime}\left(x^{\omega}\right)\right]>1$ and therefore $\gamma_{\mathrm{I}}<0$. Thus we reach a contradiction, in the sense that both $w_{\mathrm{I}}=0$ and $\mathscr{A}(v)=0$ cannot be true. Since $\mathscr{A}(v)$ cannot be negative by definition, if $w_{\mathrm{I}}=0$ the only possibility is that $\mathscr{A}(v)>0$.

Proof of Proposition 2. (The proof works in the same manner as the proof of Proposition 1 and we therefore only sketch the argument.) Consider the FOC for the maximization program in (9). Let $\gamma_{u}, \gamma_{\mathrm{I}}, \gamma_{\mathrm{N}}$ be the Lagrange multipliers associated with the constraints (i), (ii), (iii).

(a) We look at the FOC at $w_{\mathrm{I}}=0$. At this point, we know that $w_{\mathrm{N}}$ has to be strictly positive to satisfy the participation constraint (i), and hence the associated multiplier, $\gamma_{\mathrm{N}}$, is zero. Thus, the FOC yield

$$
\left\{\begin{array}{l}
\gamma_{u}=\frac{\mathrm{E}_{m \times n}\left[1 / \lambda^{\omega} p_{y}\right]}{\mathrm{E}_{\underline{\mu} \times \bar{v}}\left[\left(1 / \lambda^{\omega} p_{x}\right) u^{\prime}\left(x^{\omega}\right)\right]} \\
\gamma_{\mathrm{I}}=-\gamma_{u} \mathrm{E}_{\underline{\mu} \times \bar{v}}\left[\frac{p_{x}+p_{y}+p_{z}^{\omega}}{p_{x}} u^{\prime}\left(x^{\omega}\right)\right]+\mathrm{E}_{m \times n}\left[\frac{\left(p_{x}+p_{y}+p_{z}^{\omega}\right)}{p_{y}}\right] .
\end{array}\right.
$$

We now study the sign of $\gamma_{\mathrm{I}}$. Proceeding as in the proof of proposition 1 it can be seen that the sign of $\gamma_{\mathrm{I}}$ (when $\left.w_{\mathrm{I}}=0\right)$ is the same as that of (17), below:

$$
-\frac{\mathrm{E}_{m \times n}\left[1 / \lambda^{\omega}\right] \mathrm{E}_{\underline{\mu} \times \bar{v}}\left[u^{\prime}\left(x^{\omega}\right)\right]}{\mathrm{E}_{\underline{\mu} \times \bar{v}}\left[\left(1 / \lambda^{\omega}\right) u^{\prime}\left(x^{\omega}\right)\right]}+\frac{\mathrm{E}_{m \times n}\left[\left(p_{x}+p_{y}+p_{z}^{\omega}\right)\right]}{\mathrm{E}_{\underline{\mu} \times \bar{v}}\left[p_{x}+p_{y}+p_{z}^{\omega}\right]} .
$$


Now, observe that if $\lambda$ is constant, i.e., $\lambda_{\mathrm{L}}=\lambda_{\mathrm{H}}$, we get that

$$
\frac{\mathrm{E}_{m \times n}\left[1 / \lambda^{\omega}\right] \mathrm{E}_{\underline{\mu} \times \bar{v}}\left[u^{\prime}\left(x^{\omega}\right)\right]}{\mathrm{E}_{\underline{\mu} \times \bar{v}}\left[\left(1 / \lambda^{\omega}\right) u^{\prime}\left(x^{\omega}\right)\right]}=1 .
$$

Furthermore,

$$
\begin{aligned}
\frac{\mathrm{E}_{m \times n}\left[\left(p_{x}+p_{y}+p_{z}^{\omega}\right)\right]}{\mathrm{E}_{\underline{\mu} \times \bar{v}}\left[p_{x}+p_{y}+p_{z}^{\omega}\right]} & =\frac{p_{x}+p_{y}+n p_{z}^{1}+(1-n) p_{z}^{\mathrm{h}}}{p_{x}+p_{y}+(1-\bar{v}) p_{z}^{1}+\bar{v} p_{z}^{\mathrm{h}}} \\
& =\frac{p_{x}+p_{y}+n p_{z}^{1}+(1-n) p_{z}^{\mathrm{h}}}{p_{x}+p_{y}+(1-(1-\mathscr{A}) n) p_{z}^{1}+(1-\mathscr{A}) n p_{z}^{\mathrm{h}}} .
\end{aligned}
$$

Hence, if $\mathscr{A}=1$, then,

$$
\frac{\mathrm{E}_{m \times n}\left[\left(p_{x}+p_{y}+p_{z}^{\omega}\right)\right]}{\mathrm{E}_{\underline{\mu} \times \bar{v}}\left[p_{x}+p_{y}+p_{z}^{\omega}\right]}=\frac{p_{x}+p_{y}+n p_{z}^{1}+(1-n) p_{z}^{\mathrm{h}}}{p_{x}+p_{y}+p_{z}^{1}}>1 .
$$

(since by assumption $n$ is different from one).

So, in the extreme case in which $\lambda_{\mathrm{L}}=\lambda_{\mathrm{H}}$ and $\mathscr{A}=1$,

$$
\begin{gathered}
-\frac{\mathrm{E}_{m \times n}\left[1 / \lambda^{\omega}\right] \mathrm{E}_{\underline{\mu} \times \bar{v}}\left[u^{\prime}\left(x^{\omega}\right)\right]}{\mathrm{E}_{\underline{\mu} \times \bar{v}}\left[\left(1 / \lambda^{\omega}\right) u^{\prime}\left(x^{\omega}\right)\right]}+\frac{\mathrm{E}_{m \times n}\left[\left(p_{x}+p_{y}+p_{z}^{\omega}\right)\right]}{\mathrm{E}_{\underline{\mu} \times \bar{v}}\left[p_{x}+p_{y}+p_{z}^{\omega}\right]} \\
=-1+\frac{\mathrm{E}_{m \times n}\left[\left(p_{x}+p_{y}+p_{z}^{\omega}\right)\right]}{\mathrm{E}_{\underline{\mu} \times \bar{v}}\left[p_{x}+p_{y}+p_{z}^{\omega}\right]}>0 .
\end{gathered}
$$

As a result, in this extreme case, the FOC are satisfied at $w_{\mathrm{I}}=0$ and $w_{\mathrm{N}}$ such that the participation constraint of the employee is just met, i.e., for these values of wages, we can find non-negative Lagrange multipliers such that the FOC of the maximization problem are satisfied. Now, by continuity, the expression on the 1.h.s. of (18) remains positive whenever $\lambda_{L}$ is close to $\lambda_{\mathrm{H}}$ and $\bar{A}<\mathscr{A}<1$, for $\overline{\mathscr{A}}$ close to 1 .

Given that $u(\cdot)$ is strictly concave, the programming problem (9) admits a unique solution. Hence, since we exhibited a solution to (9) at which $w_{\mathrm{I}}=0$, the assertion in part (a) of Proposition 2 follows.

(b) Let us suppose that $w_{\mathrm{I}}=0$ and $\mathscr{A}=0$, that is, $\mu$ and $v$ are now two probability distributions and, more precisely, $\mu=m$ and $v=n$. Now, following on from (17), substituting in $n$ in place of $\bar{v}$ and $m$ in place of $\underline{\mu}$, we see that the sign of $\gamma_{\mathrm{I}}$ is the same as that of the expression below

$$
\begin{aligned}
- & \frac{\mathrm{E}_{m \times n}\left[1 / \lambda^{\omega}\right] \mathrm{E}_{m \times n}\left[u^{\prime}\left(x^{\omega}\right)\right]}{\mathrm{E}_{m \times n}\left[\left(1 / \lambda^{\omega}\right) u^{\prime}\left(x^{\omega}\right)\right]}+\frac{\mathrm{E}_{m \times n}\left[\left(p_{x}+p_{y}+p_{z}^{\omega}\right)\right]}{\mathrm{E}_{m \times n}\left[p_{x}+p_{y}+p_{z}^{\omega}\right]} \\
=- & -\frac{\mathrm{E}_{m \times n}\left[1 / \lambda^{\omega}\right] \mathrm{E}_{m \times n}\left[u^{\prime}\left(x^{\omega}\right)\right]}{\mathrm{E}_{m \times n}\left[\left(1 / \lambda^{\omega}\right) u^{\prime}\left(x^{\omega}\right)\right]}+1,
\end{aligned}
$$

Following the same argument as in the proof of (b) of proposition 1, it can be shown that both $w_{\mathrm{I}}=0$ and $\mathscr{A}=0$ cannot be true, since it would otherwise imply that $\gamma_{\mathrm{I}}$ is negative. Hence, if $w_{\mathrm{I}}=0$ the only possibility is that $\mathscr{A}>0$. 


\section{References}

Camerer, C., 1995. Individual Decision Making. In: Kagel, J.K., Roth, A. (Eds.), Handbook of Experimental Economics, Princeton University Press, Princeton, NJ.

Card, D., 1986. An empirical model of wage indexation provisions in union contracts. Journal of Political Economy 94 (3, part 2), S144-S175.

Dow, J., Werlang, S., 1992. Uncertainty aversion, risk aversion, and the optimal choice of portfolio. Econometrica 60 (1), 197-204.

Ellsberg, D., 1961. Risk, ambiguity, and the savage axioms. Quarterly Journal of Economics 75, 643-669.

Epstein, L., Wang, T., 1994. Intertemporal asset pricing under Knightian uncertainty. Econometrica 62 (3), 283-322.

Farber, H.S., 1978. Individual preferences and union wage determination: the case of the united mine workers. Journal of Political Economy 86, 923-942.

Fischer, S., 1977. Wage indexation and macroeconomic stability. In: Brunner, K., Meltzer, A.H. (Eds.), Stabilization of the Domestic and International Economy, Vol. 5, Amsterdam. Carnegie-Rochester Conference Series on Public Policy, North-Holland, Amsterdam.

Gilboa, I., Schmeidler, S., 1989. Maxmin expected utility with a non-unique prior. Journal of Mathematical Economics 18, 141-153.

Gray, J.A., 1976. Wage indexation: a macroeconomic approach. Journal of Monetary Economics 2, 221-235.

Gray, J.A., 1978. On indexation and contract length. Journal of Political Economy 86 (1), 1-18.

Hansen, L.P., Sargent, T.J., Tallarini Jr., T.D., 1999. Robust permanent income and pricing. The Review of Economic Studies 66, 873-907.

Hansen, L.P., Sargent, T.J., Turmuhambetova, G., Williams, N., 2001. Robustness and Uncertainty Aversion, Discussion paper, University of Chicago.

Holland, A.S., 1995. Inflation and wage Indexation in the postwar United States. The Review of Economics and Statistics 77 (1), 172-176.

Jovanovic, B., Ueda, M., 1997. Contracts and money. Journal of Political Economy 105, 700-708.

Mukerji, S., 1997. Understanding the nonadditive probability decision model. Economic Theory 9 (1), $23-46$.

Mukerji, S., 1998. Ambiguity aversion and incompleteness of contractual form. American Economic Review 88 (5), 1207-1231.

Mukerji, S., Tallon, J.-M., 2004. Ambiguity aversion and the absence of indexed debt. Economic Theory, forthcoming.

Mukerji, S., Tallon, J.-M., 2001. Ambiguity aversion and incompleteness of financial markets. Review of Economic Studies 68 (4), 883-904.

Savage, L., 1954. The Foundations of Statistics. Wiley, New York.

Schmeidler, D., 1989. Subjective probability and expected utility without additivity. Econometrica 57 (3), 571-587.

Shiller, R.J., 1997. Public resistance to indexation: a puzzle. Brookings Papers on Economic Activity 1, $159-226$.

Tallon, J.-M., 1998. Asymmetric information, nonadditive expected utility and the information revealed by prices: an example. International Economic Review 39 (2), 329-342. 